\title{
RoundTrip Ontology Authoring
}

\author{
Brian Davis ${ }^{1}$, Ahmad Ali Iqbal ${ }^{1,3}$, Adam Funk ${ }^{2}$, Valentin Tablan ${ }^{2}$, \\ Kalina Bontcheva ${ }^{2}$, Hamish Cunningham ${ }^{2}$, and Siegfried Handschuh ${ }^{1}$ \\ ${ }^{1}$ Digital Enterprise Research Institute, Galway, Ireland \\ ${ }^{2}$ University of Sheffield, UK \\ ${ }^{3}$ University of New South Wales, Australia
}

\begin{abstract}
Controlled Language (CL) for Ontology Editing tools offer an attractive alternative for naive users wishing to create ontologies, but they are still required to spend time learning the correct syntactic structures and vocabulary in order to use the Controlled Language properly. This paper extends previous work (CLOnE) which uses standard NLP tools to process the language and manipulate an ontology. Here we also generate text in the CL from an existing ontology using template-based (or shallow) Natural Language Generation (NLG). The text generator and the CLOnE authoring process combine to form a RoundTrip Ontology Authoring environment: one can start with an existing imported ontology or one originally produced using CLOnE, (re)produce the Controlled Language, modify or edit the text as required and then turn the text back into the ontology in the CLOnE environment. Building on previous methodology we undertook an evaluation, comparing the RoundTrip Ontology Authoring process with a well-known ontology editor; where previous work required a CL reference manual with several examples in order to use the controlled language, the use of NLG reduces this learning curve for users and improves on existing results for basic ontology editing tasks.
\end{abstract}

\section{Introduction}

Formal data representation can be a significant deterrent for non-expert users or small organisations seeking to create ontologies and subsequently benefit from adopting semantic technologies. Existing ontology authoring tools such as Protég 11 attempt to resolve this, but they often require specialist skills in ontology engineering on the part of the user. This is even more exasperating for domain specialists, such as clinicians, business analysts, legal experts, etc. Such professionals cannot be expected to train themselves to comprehend Semantic Web formalisms and the process of knowledge gathering; involving both a domain expert and an ontology engineer can be time-consuming and costly. Controlled languages for knowledge creation and management offer an attractive alternative for naive users wishing to develop small to medium sized ontologies or a first draft ontology which can subsequently post-edited by the Ontology

\footnotetext{
1 http://protege.stanford.edu
} 
Engineer. In previous work[1], we presented CLOnE - Controlled Language for Ontology Editing which allows naive users to design, create, and manage information spaces without knowledge of complicated standards (such as XML, RDF and OWL) or ontology engineering tools. CLOnE's components are based on GATE's existing tools for IE (Information Extraction) and NLP (Natural Language Processing) [2].

The CLOnE system was evaluated using a repeated-measures, task-based methodology in comparison with a standard ontology editor - Protégé. CLOnE performed favourably with test users in comparison to Protégé. Despite the benefits of applying Controlled Language Technology to Ontology Engineering, a frequent criticism against its adoption, is the learning curve associated with following the correct syntactic structures and/or terminology in order to use the Controlled Language properly. Adhering to a controlled language can be, for some naive users, time consuming and annoying. These difficulties are related to the habitability problem, whereby users do not really know what commands they can or cannot specify to the NLI (Natural Language Interface) 3]. Where the CLOnE system uses natural language analysis to unambiguously parse CLOnE in order to create and populate an ontology, the reverse of this process, NLG (Natural Language Generation), involves the generation of the CLOnE language from an existing ontology. The text generator and CLOnE authoring processes combine to form a RoundTrip Ontology Authoring(ROA) environment: a user can start with an existing imported ontology or one originally produced using CLOnE, (re)produce the Controlled Language using the text generator, modify or edit the text as required and subsequently parse the text back into the ontology using the CLOnE environment. The process can be repeated as necessary until the required result is obtained. Building on previous methodology [1], we undertook a repeated-measures, task-based evaluation, comparing the RoundTrip Ontology Authoring process with Protégé. Where previous work required a reference guide in order to use the controlled language, the substitution of NLG can reduce the learning curve for users, while simultaneously improving upon existing results for basic Ontology editing tasks. The remainder of this paper is organized as follows: Section 2 discusses the design and implementation of the ROA pipeline focusing on the NLG component - the ROA text generator, Section 3 presents our evaluation and discusses our quantitative findings. Section 4 discusses related work. Finally, Section 5 and Section 6 offer conclusions and future work.

\section{Design and Implementation}

In this section, we describe the overall architecture of the Round Trip Ontology Authoring (ROA) pipeline which is implemented in GATE [2. We discuss briefly extensions to existing CLOnE components of ROA, but focus the attention of this section towards describing the CLOnE text generator, the algorithm used and the XML configuration file containing templates needed to configure the controlled language output of the generator. 


\subsection{RoundTrip Ontology Authoring (ROA) and CLOnE}

ROA builds on and extends the existing advantages of the CLOnE software and input language, which are described below:

1. ROA requires only one interpreter or runtime environment, the Java 1.6 JRE.

2. ROA like CLOnE uses a sub-language of English.

3. As far as possible, CLOnE is grammatically lax; in particular it does not matter whether the input is singular or plural (or even in grammatical agreement).

4. ROA can be compact; the user can create any number of classes or instances in one sentence.

5. ROA is more flexible and easier to learn by using simple examples of how to edit the controlled language generated by the text generator in order to modify the Ontology. It reduces the need to learn the Controlled Language by following examples, style guides or CLOnE syntactic rules. Instead, a user can create or modify various classes and instances in one (generated) sentence or (using simple copy and paste) create new properties between new or existing classes and instances.

6. The CLOnE grammar within ROA has been extended to handle simple verbs and phrasal verbs.

7. Like CLOnE any valid sentence of ROA can be unambiguously parsed.

8. The advantage of the GATE Ontology API allows users to import existing Ontologies for generation, subsequent editing in ROA and export the result to different Ontology formats.

9. SimpleNLG2 2 has been added into the ROA text generator to lexicalize unseen properties.

Procedurally, CLOnE's analysis consists of the ROA pipeline of processing resources (PRs) shown in Figure 1 (left dotted box). This pipeline starts with a series of fairly standard GATE NLP tools which add linguistic annotations and annotation features to the document. These are followed by three PRs developed particularly for CLOnE: the gazetteer of keywords and phrases fixed in the controlled language and two JAPE 3 transducers which identify quoted and unquoted chunks. Names enclosed in pairs of single or double quotation marks can include reserved words, punctuation, prepositions and determiners, which are excluded from unquoted chunks in order to keep the syntax unambiguous. The last stage of analysis, the CLOnE JAPE transducer, refers to the existing ontology in several ways in order to interpret the input sentences. Table 1 below provides an excerpt of the grammar rules of the CLOnE language. We refer the reader to [14] for additional rules and examples.

\footnotetext{
2 http://www.csd.abdn.ac.uk/ ereiter/simplenlg/

3 GATE provides the JAPE (Java Annotation Pattern Engine) language for matching regular expressions over annotations, adding additional annotations to matched spans, and manipulating the match patterns with Java code.
} 


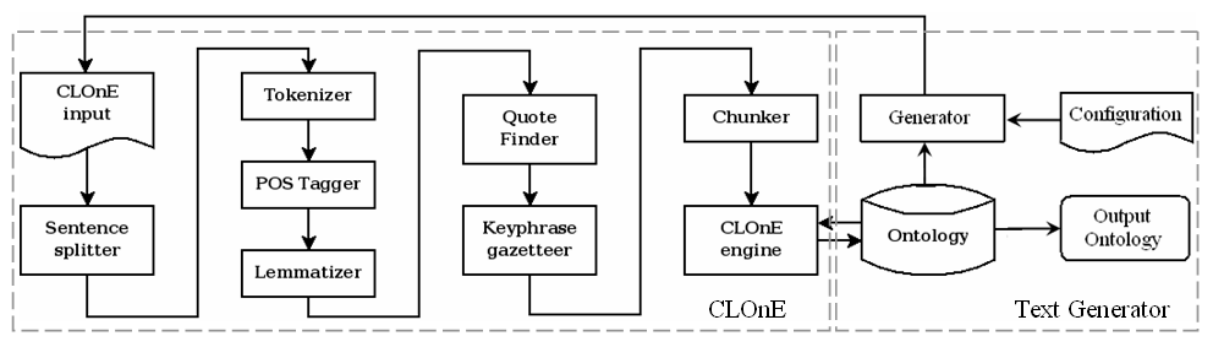

Fig. 1. The ROA RoundTrip Ontology Authoring pipeline

Table 1. Excerpt of CLOnE grammar with examples

\begin{tabular}{|c|c|c|}
\hline Sentence Pattern & Example & Usage \\
\hline Forget everything. & Forget everything. & $\begin{array}{l}\text { Clear the whole ontology } \\
\text { corpus to start with the } \\
\text { new ontology. }\end{array}$ \\
\hline $\begin{array}{l}\text { (Forget that) There is/are } \\
<\text { CLASSES }>\text {. }\end{array}$ & $\begin{array}{l}\text { There are researchers, } \\
\text { universities and } \\
\text { conferences. }\end{array}$ & $\begin{array}{l}\text { Create or delete (new) } \\
\text { classes. }\end{array}$ \\
\hline $\begin{array}{lcr}\text { (Forget } & \text { that) } & <\text { IN- } \\
\text { STANCES }>\text { is } & \text { a } / \text { are } \\
<\text { CLASS }>\text {. } & & \end{array}$ & $\begin{array}{l}\text { Ahmad Ali Iqbal and } \\
\text { Brian Davis are 'Ph.D. } \\
\text { Scholar'. }\end{array}$ & $\begin{array}{l}\text { Create (or delete) in- } \\
\text { stances of the class. }\end{array}$ \\
\hline $\begin{array}{l}\text { (Forget that) }<\text { SUB- } \\
\text { CLASSES }>\text { is/are a } \\
\text { type/types of <SUPER- } \\
\text { CLASS }>\text {. }\end{array}$ & $\begin{array}{l}\text { 'Ph.D. Scholar' is a } \\
\text { type of Student. }\end{array}$ & $\begin{array}{l}\text { Make subclass(es) of an } \\
\text { existing super-class. 'For- } \\
\text { get that' only unlinks the } \\
\text { the subclass-superclass } \\
\text { relationship. }\end{array}$ \\
\hline $\begin{array}{lr}\text { Forget that) } & <\text { CLASSES } / \\
\text { INSTANCES }> & <\text { VERB } \\
\text { PROPERTY }> & <\text { CLASSES } / \\
\text { INSTANCES }>\text {. } & \end{array}$ & $\begin{array}{l}\text { Professor supervises } \\
\text { student. }\end{array}$ & $\begin{array}{l}\text { Create the property of the } \\
\text { form Domain_verb_Range } \\
\text { either between two classes } \\
\text { or instances. }\end{array}$ \\
\hline
\end{tabular}

\subsection{Text Generation of CLOnE}

The text generation component in Figure 1 (right dotted box) displayed in the ROA pipeline is essentially an Ontology Verbalizer. Unlike some NLG systems, the communicative goal of the text generator is not to construct tailored reports for specific content within the knowledge base or to respond to user specific queries. Hence no specific content selection subtask or "choice" is performed since our goal is to describe and present the Ontology in textual form as unambiguous subset of English - the CLOnE language for reading, editing and amendment. We select the following content from the Ontology: top level classes, subclasses, instances, class properties, their respective domain and ranges and instance properties. The text generator is configured using an XML file, whereby text templates are instantiated and filled by the values from the Ontology. This 
since the triples are matched in a top down fashion according to their textual ordering. An example of referencing can be seen in line 188 of the out element of the template shown in Figure 2 for generating class properties.

In Stage 3 the out section of the template describes how text is generated from a successful match. It contains phrase templates that have text elements and references to values matched within the in elements. Phrases are divided into singular and plural forms. Plural variants are executed when several triples are grouped together to generate a single sentence (Sentence Aggregation) based on a list of Ontology objects (i.e., There are Conferences, Students and Universities). Text elements within a template are simply copied into the output while reference values are replaced with actual values based on matching triple specifications. We also added a small degree of lexicalization into the Text Generator PR, whereby, for example, an unseen property, which is treated as a verb is inflected correctly for surface realisation i.e. study and studies. This involves a small amount of dictionary look-up using the SimpleNLG Library to obtain the third person singular inflection studies from study to produce Brian Davis studies at NUIG. The out elements of the generation template also provide several phrase templates for the singular and plural sections. These are applied in rotation to prevent tedious and repetitious output.

Stage 2 also groups matches together into sets that can be expressed together in a plural form. For this to proceed, the required condition is that the difference between matches, occurs in only one of the references used in the phrase templates, i.e., if singular variants would only differ by one value. A specialized generation template with no in restrictions is also included in the configuration file. This allows for the production of text where there are no specific input triple dependencies.

\section{Evaluation}

\subsection{Methodology}

Our methodology is deliberately based on the criteria previously used to evaluate CLOnE 114, so that we can fairly compare the earlier results using the CLOnE software with the newer RoundTrip Ontology Authoring(ROA) process. The methodology involves a repeated-measures, task-based evaluation: each subject carries out a similar list of tasks on both tools being compared. Unlike our previous experiment, the CLOnE reference guide list and examples are withheld from the test users, so that we can measure the benefits of substituting the text generator for the reference guide and determine its impact on the learning process and usability of CLOnE. Furthermore, we used a larger sample size and more controls for bias. All evaluation material and data are available online for inspection, including the CLOnE evaluation results for comparisond. The evaluation contained the following:

\footnotetext{
4 http://smile.deri.ie/evaluation/2008/ROA
} 
- A pre-test questionnaire asking each subject to test their degree of knowledge with respect to ontologies, the Semantic Web, Protégé and Controlled Languages. It was scored by assigning each answer a value from 0 to 2 and scaling the total to obtain a score of $0-100$.

- A short document introducing Ontologies, the same 'quick start' Protégé instructions as used in [4] (partly inspired by Protégé's Ontology 101 documentation [5]), and an example of editing CLOnE text derived from the text generator. The CLOnE reference guide and detailed grammar examples used in for the previous experiment 4] were withheld. Subjects were allowed to refer to an example of how to edit generated Controlled Language but did not have access to CLOnE reference guide.

- A post-test questionnaire for each tool, based on the System Usability Scale (SUS), which also produces a score of 0-100 to compare with previous results [6].

- A comparative questionnaire similar to the one used in 4] was applied to measure each user's preference for one of the two tools. It is scored similarly to SUS so that 0 would indicate a total preference for Protégé, 100 would indicate a total preference for ROA, and 50 would result from marking all the questions neutral. Subjects were also given the opportunity to make comments and suggestions.

- Two equivalent lists of ontology-editing tasks, each consisting of the following subtasks:

- creating two subclasses of existing classes,

- creating two instances of different classes, and

- either (A) creating a property between two classes and defining a property between two instances, or (B) extending properties between two pairs of instances.

For both task lists, an initial ontology was created using CLOnE. The same ontology was loaded into Protégé for both tasks and the text generator was executed to provide a textual representation of the ontology for editing purposes(see Figure 3), again for both tasks.

For example, Task List A is as follows.

- Create a subclass Institute of University.

- Create a subclass Workshop of Conference.

- Create an instance International Semantic Web Conference of class Conference.

- Create an instance DERI of class Institute.

- Create a property that Senior Researchers supervise Student.

- Define a property that Siegfried Handschuh supervises Brian Davis.

\subsection{Sample Quality}

We recruited 20 volunteers from the Digital Enterprise Research Institute, Galway 5 . The sample size $(n=20)$ satisfies the requirements for reliable SUS

\footnotetext{
5 http://www.deri.ie
} 


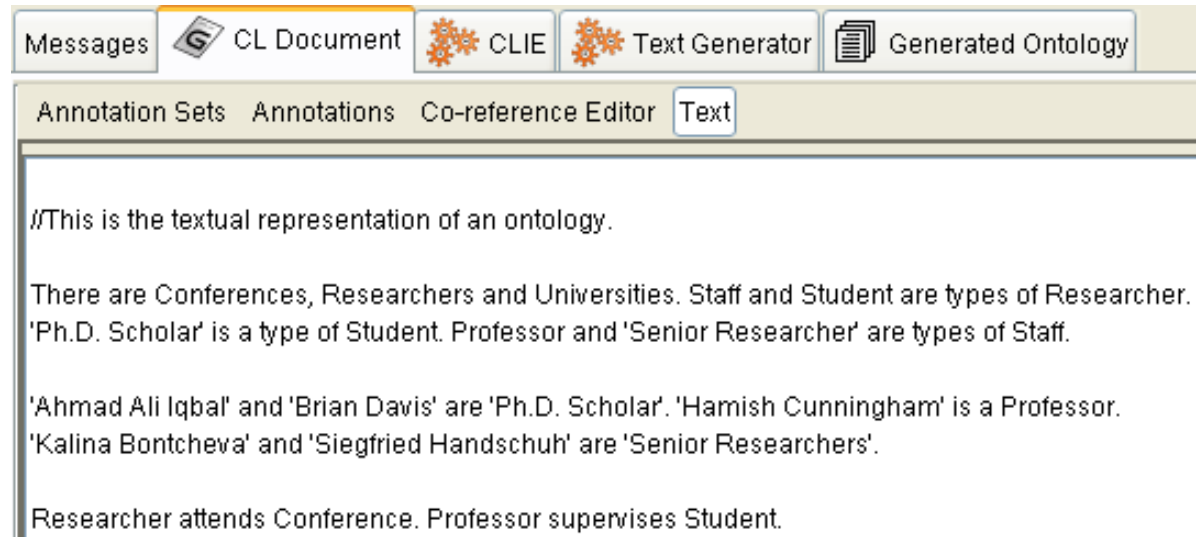

Fig. 3. Text Generated by ROA

evaluations [7]. We recruited subjects with an industrial background (I) and participants with a research background $(\mathbf{R})$. See (in Table 5 ) for details. In addition we attempted to control bias by selecting volunteers who were either:

- Research Assistants/Programmers/Post-Doctoral Researchers with an industrial background either returning (or new) to Academic Research respec$\operatorname{tively}(\mathbf{I})$,

- Postgraduate Students who were new to the Semantic Web and unfamiliar with Ontology Engineering $(\mathbf{R})$,

- Researchers from the E-learning and Sensor Networks lab but not from the Semantic Web Cluster $(\mathbf{R})$,

- Researchers with no background in Natural Language Processing or Ontology Engineering $(\mathbf{R})$ or

- Industrial Collaborators (I).

In all cases, we tried to ensure that participants had limited or no knowledge of GATE or Protégé. First, subjects were asked to complete the pre-test questionnaire, then they were permitted time to read the Protégé manual and Text Generator examples, and lastly they were asked to carry out each of the two task lists with one of the two tools. (Half the users carried out task list A with ROA and then task list B with Protégé; the others carried out A with Protégé and then B with ROA.) Each user's time for each task list was recorded. After each task list the user completed the SUS questionnaire for the specific tool used, and finally the comparative questionnaire. Comments and feedback were also recorded on the questionnaire forms.

\subsection{Quantitative Findings}

Table 2 summarizes the main measures obtained from our evaluation. We used SPS\$6 to generate all our statistical results. In particular the mean ROA SUS

${ }^{6}$ SPSS 2.0, http://www.spss.com 
Table 2. Summary of the questionnaire scores

\begin{tabular}{lccrr}
\hline Measure & \multicolumn{4}{c}{ min mean median } \\
\hline Pre-test scores & 17 & 42 & 42 & 75 \\
ROA SUS rating & 48 & 74 & 70 & 100 \\
Protégé SUS rating & 10 & 41 & 41 & 85 \\
R/P Preference & 40 & 72 & 79 & 95 \\
\hline
\end{tabular}

Table 3. Confidence intervals (95\%) for the SUS scores

\begin{tabular}{lccc}
\hline Tool & \multicolumn{3}{c}{ Confidence intervals } \\
& Task list A & Task list & B Combined \\
\hline Protégé & $28-55$ & $29-51$ & $32-49$ \\
ROA & $63-77$ & $69-84$ & $68-79$ \\
\hline
\end{tabular}

Table 4. Correlation coefficients

\begin{tabular}{llrrr}
\hline Measure & Measure & Pearson's Spearman's Correlation \\
\hline Pre-test & ROA time & -0.41 & -0.21 & weak - \\
Pre-test & Protégé time & -0.28 & -0.35 & none \\
Pre-test & ROA SUS & -0.02 & -0.00 & none \\
Pre-test & Protégé SUS & -0.32 & -0.29 & weak - \\
\hline ROA time & Protégé time & 0.53 & 0.58 & + \\
ROA time & ROA SUS & -0.65 & -0.52 & - \\
Protégé time Protégé SUS & 0.53 & 0.56 & + \\
ROA time & Protégé SUS & -0.14 & -0.10 & none \\
Protégé time ROA SUS & -0.02 & -0.09 & none \\
\hline ROA SUS & Protégé SUS & 0.04 & -0.01 & none \\
ROA SUS & R/P Preference & 0.58 & 0.56 & + \\
Protégé SUS R/P Preference & -0.01 & 0.10 & none \\
\hline
\end{tabular}

score is above the baseline of $65-70 \%$ while the mean SUS score for Protégé is well below the baseline [8]. In the ROA/Protégé Preference (R/P Preference) scores, based on the comparative questionnaires, we note that the scores also favour on average ROA over Protégé. Confidence intervals are displayed in Table 37

We also generated Pearson's and Spearman's correlations coefficients 910]. Table 4 displays the coefficients. In particular, we note the following results.

- The pre-test score has a weak negative correlations the with ROA task time.

- There are no correlations with pre-test score and the ROA SUS score.

- The pre-test score has a weak negative correlation with the Protégé SUS score.

- There are no correlations with pre-test score and the Protégé time.

\footnotetext{
${ }^{7}$ A data sample's $95 \%$ confidence interval is a range $95 \%$ likely to contain the mean of the whole population that the sample represents 9 .
} 
- In previous results in comparing CLOnE and Protégé, the task times for both tools were more positively correlated with each other while in the case of ROA and Protégé, there correlation has being weakened by a significant $32 \%$ of its original value (of $78 \%$ ) reported for CLOnE [1, indicating that the users tended not spend the equivalent time completing both ROA and Protégé tasks.

- There is a moderate correlation with Protégé task time and Protégé SUS scores.

- There is a strong negative correlation of -0.65 between the ROA task time and the ROA SUS scores. Our previous work reported no correlation between the CLOnE task time and CLOnE SUS time. A strong negative or inverse correlation implies that users who spent less time completing a task using ROA tended to produce high usability scores - favouring ROA. More importantly, we noted that the associated probability reported by SPSS, was less then the typical 5\% cut-off point used in social sciences. This implies there is a $5 \%$ chance that the true population coefficient is very unlikely to be 0 (no relationship). Conversely, one can infer statistically that for 19 out of 20 (95\%)users, with little or no experience in either NLP or Protégé who favour RoundTrip Ontology Authoring over Protégé also tend to spend less time completing Ontology editing tasks.

- The R/P Preference score correlates moderately with the ROA SUS score, similar to previous results, but no longer retains a significant inverse correlation with the Protégé SUS score. The reader should note the R/P Preference scores favour ROA over Protégé.

We also varied the tool order evenly among our sample. As noted previously in [1, once again the SUS scores have differed slightly according to tool order (as indicated in Table 3). Previous SUS scores for Protégé tended to be slightly lower for B than for A, which we believe may have resulted from the subjects' decrease in interest as the evaluation progressed. While in previous results there was a decrease in SUS scores for CLOnE (yet still well above the SUS baseline), in the case of ROA however, the SUS scores increased for task B (see Table 3), implying that if waning interest was a factor in the decrease in SUS scores for CLOnE, it does not appear to be the case for ROA. What is of additional interest is that group I, subjects with industrial background scored on average 10\% higher for both ROA SUS and ROA/Protégé, which implies that Industrial collaborators or professionals with an Industrial background favoured a natural language interface over a standard Ontology Editor even more than Researchers.

\subsection{User Feedback}

The test users also provided several suggestions/comments about ROA.

- "RoundTrip Ontology Authoring becomes much easier, once the rules are learnt". (This is very interesting considering that no syntax rules, extended examples or restricted vocabulary list were provided). 
- Use of inverted commas should be used only once and afterwards, if same the class /instance is reused, the system should automatically recognise it as the previous word.

- Many users suggested displaying the ontology pane on the right hand side of the text pane, where test users edit the text instead of moving between two separate panes.

- Some users suggested dynamic ontology generation, once a user finishes typing a sentence, the changes should be displayed automatically in the ontology pane.

- Similar suggestions to the previous evaluation were provided for user autocompletion, syntax highlighting, options about available classes, instances or property names and keywords should be displayed, a similar concept to modern Word Processor or programming IDEs such as eclipse.

- Some test users with an industrial background demonstrated concern regarding scalability and ROA using with a larger business related ontology and suggest capabilities for verbalizing a portion of the ontology tree within the Ontology viewer, using text generation for subsequent editing.

- Some test users appreciated the singular/plural forms and sentence handling of ROA (e.g., study, studies).

Table 5. Groups of subjects by source and tool order

\begin{tabular}{lrrr}
\hline Source & \multicolumn{3}{c}{ Tool order Total } \\
& $\mathrm{PR}$ & $\mathrm{RP}$ & \\
\hline R Researcher & 5 & 7 & 12 \\
I Industry & 5 & 3 & 8 \\
\hline Total & 10 & 10 & 20 \\
\hline
\end{tabular}

Table 6. Comparison of the two sources of subjects

\begin{tabular}{llllrr}
\hline Measure & \multicolumn{6}{l}{ Group } & min & mean & median & max \\
\hline Pre-test & $\mathrm{R}$ & 17 & 38 & 38 & 58 \\
& $\mathrm{I}$ & 17 & 47 & 50 & 75 \\
\hline ROA SUS & $\mathrm{R}$ & 48 & 69 & 70 & 82 \\
& $\mathrm{I}$ & 65 & 80 & 80 & 100 \\
\hline Protégé SUS & $\mathrm{R}$ & 10 & 30 & 28 & 52 \\
& $\mathrm{I}$ & 12 & 48 & 49 & 85 \\
\hline R/P Preference $\mathrm{R}$ & 40 & 68 & 72 & 88 \\
& $\mathrm{I}$ & 65 & 78 & 78 & 95 \\
\hline
\end{tabular}

\section{Related Work}

"Controlled Natural Languages (CL)s are subsets of natural language whose grammars and dictionaries have been restricted in order to reduce or eliminate both ambiguity and complexity" 11. CLs were later developed specifically for 
computational treatment and have subsequently evolved into many variations and flavours such as Smart's Plain English Program (PEP), White's International Language for Serving and Maintenance (ILSAM) 12 and Simplified English 8 They have also found favour in large multi-national corporations, usually within the context of machine translation and machine-aided translation of user documentation [1112.

The application of CLs for ontology authoring and instance population is an active research area. Attempto Controlled English 9 (ACE) [13], is a popular CL for ontology authoring. It is a subset of standard English designed for knowledge representation and technical specifications, and is constrained to be unambiguously machine-readable into DRS - Discourse Representation Structure. ACE OWL, a sublanguage of ACE, proposes a means of writing formal, simultaneously human- and machine-readable summaries of scientific papers [1415]. Similar to RoundTrip Ontology Authoring, ACE OWL also aims to provide reversibility (translating OWL DL into ACE). The application NLG, for the purposes editing existing ACE text, is mentioned in [16]. The paper discusses the implementation of the shallow NLG system - an OWL Verbalizer, focusing primarily on the OWL to ACE rewrite rules, however no evaluation or quantitative data are provided in attempt to measure the impact of NLG in the authoring process. Furthermore OWL's allValuesFrom must be translated into a construction which can be rather difficult for humans to read. A partial implementation is however available for public testing 10 .

Another well-known implementation which employs the use of NLG to aid the knowledge creation process is WYSIWYM (What you see is what you meant). It involves direct knowledge editing with natural language directed feedback. A domain expert can edit a knowledge based reliably by interacting with natural language menu choices and the subsequently generated feedback, which can then be extended or re-edited using the menu options. The work is conceptually similar to RoundTrip Ontology Authoring, however the natural language generation occurs as a feedback to guide the user during the editing process as opposed to providing an initial summary in Controlled Language for editing. A usability evaluation is provided in 17, in the context of knowledge creation, partly based on IBM heuristic evaluation 11 , but no specific quantitative data that we are aware of, is presented. However, evaluation results are available for the MILE (Maritime Information and Legal Explanation) application, which used WYSIWYM, but in the context of query formulation for the CLIME 12 project, of which the outcome was favourable [17].

Similar to WYSIWYM is GINO (Guided Input Natural Language Ontology Editor) provides a guided, controlled NLI (natural language interface) for

\footnotetext{
8 http://www.simplifiedenglish-aecma.org/Simplified_English.htm

9 http://www.ifi.unizh.ch/attempto/

10 http://attempto.ifi.uzh.ch/site/tools/

11 http://www-03.ibm.com/able/resources/uebeforeyoubegin.html

12 CLIME, Cooperative Legal Information Management and Explanation, Esprit Project EP25414.
} 
domain-independent ontology editing for the Semantic Web. GINO incrementally parses the input not only to warn the user as soon as possible about errors but also to offer the user (through the GUI) suggested completions of words and sentences - similarly to the "code assist" feature of Eclips 13 and other development environments. GINO translates the completed sentence into triples (for altering the ontology) or SPARQL ${ }^{14}$ queries and passes them to the Jena Semantic Web framework. Although the guided interface facilitates input, the sentences are quite verbose and do not allow for aggregation. A full textual description of the Ontology is not realized as is the case of the CLOnE text generator [18. Furthermore, similar, to our evaluation, a small usability evaluation was conducted using SUS [6], however the sample set of six was too small to infer any statistically significant results 7 . In addition, GINO was not compared to any existing Ontology editor during the evaluation. Finally, [19] presents an Ontology based Controlled Natural Language Editor, similar to GINO, which uses a CFG (Context-free grammar) with lexical dependencies - CFG-DL to generate RDF triples. To our knowledge the system ports only to RDF and does not cater for other Ontology languages. Furthermore no quantitative user evaluation is provided.

Other related work involves the application of Controlled Languages for Ontology or knowledge base querying, which represent a different task than that of knowledge creation and editing but are worth mentioning for completeness sake. Most notably AquaLog 15 is an ontology-driven, portable Question-Answering (QA) system designed to provide a natural language query interface to semantic mark-up stored in a knowledge base. PowerAqua [20] extends AquaLog, allowing for an open domain question-answering for the semantic web. The system dynamically locates and combines information from multiple domains.

\section{Conclusion and Discussion}

The main research goal of this paper is to assess the effect of introducing Natural Language Generation (NLG) into the CLOnE Ontology authoring process to facilitate RoundTrip Ontology Authoring. The underlying basis of our research problem is the habitability problem (See Section 1): How can we reduce the learning curve associated with Controlled Languages? And how can we ensure their uptake as a Natural Language Interface (NLI)? Our contribution is empirical evidence to support the advantages of combining of NLG with ontology authoring, a process known as RoundTrip Ontology Authoring (ROA).

The reader should note, that we compared Protégé with ROA, because Protégé is the standard tool for ontology authoring. Previous work [1 compared CLOnE with Protégé. Hence, in order to compare ROA with CLOnE, it

\footnotetext{
13 http://www .eclipse.org/

14 http://www.w3.org/TR/rdf-sparql-query/

15 http://kmi.open.ac.uk/technologies/aqualog/
} 
was necessary to repeat the experiment and use Protégé as the baseline. We make no claims that Protégé should be replaced with ROA, the point is that ROA can allow for the creation of a quick easy first draft of a complex Ontology by domain experts or the creation of small to medium sized Ontologies by novice users. Domain experts are not Ontology Engineers. Furthermore, a large percentage of an initial Ontology would naturally consists of taxonomic relations and simple properties/relations.

Our user evaluation consistently indicated that our subjects found ROA (and continue to find CLOnE ) significantly more usable and preferable than Protégé for simple Ontology editing tasks. In addition our evaluation differs, in that we implemented more tighter restrictions during our selection process, to ensure that users had no background in NLP or Ontology engineering. Furthermore, $40 \%$ of our subjects with an industrial background, tended to score ROA $10 \%$ higher then Researchers indicating that a NLI to a Ontology Editor might be a preferred option for Ontology development within industry.

In detail, this evaluation differs from previous work 1 by two important factors: (1) we excluded the CLOnE reference manual from the training material provided in the previous evaluation; and (2) we introduced a Text Generator, verbalizing CLOnE text from a given populated Ontology and asked users to edit the Ontology, using the generated CLOnE text based on an example provided. We observed two new significant improvements in our results: (1) the previous evaluation indicated a strong correlation between CLOnE task times and Protégé task times, this correlation has significantly weaken by $32 \%$ between ROA and Protégé task times. Hence, where users previously required the equivalent time to implement tasks both in CLOnE and Protégé, this is no longer the case with ROA (the difference being the text generator); and (2) our previous evaluation indicated no correlation between either CLOnE/Protégé task times and their respective SUS scores. However, with ROA, we can now infer that $95 \%$ of the total population of naive users, who favour RoundTrip Ontology Authoring over Protégé, would also tend to spend less time completing Ontology editing tasks. We suspect that this is due to the reduced learning curve caused by the text generator. Furthermore, ROA tended to retain user interest, which CLOnE did not. We suspect that the absence of the need to refer to the CL reference guide was a factor in this. While Protégé is intended for more sophisticated knowledge engineering work, this is not the case for ROA. Scalability, both in performance and usage, was also an issue raised by our test subjects. From a performance perspective, when loading large Ontologies, we do not forsee any major issues as ROA is currently being ported to the newest release of GATE which contains a completely new Ontology API that utilises the power of OWLIM - OWL in Memory, a high performance semantic repository developed at Ontotext 16 . Finally, from a user perspective, authoring memory frequently used in translation memory systems or text generation of selective portions of the Ontology (using a Visual Resource) could significantly aid the navigation and authoring of large Ontologies.

16 http://www. ontotext.com/owlim/ 


\section{Continuing and Future Work}

Several interesting and useful suggestions for improvements to ROA were made, many of which were already under development within the Nepomul 17 (The Social Semantic Desktop) project. ROA has been ported to a Nepomuk-KDE 18 application, Semr 19 for Semantic Notetaking and will be also be targeted towards the task of semi-automatic semantic annotation. Furthermore, the ROA text generator was recently used in KnowledgeWeh20 for the verbalization of suggestions for semi-automatic ontology integration. Finally, ROA is being applied within the EPSRC-funded Easy project to create a controlled natural language interface for editing IT authorization policies (access to network resources such as directories and printers) stored as Ontologies.

\section{Acknowledgements}

This research has been partially supported by the following grants: KnowledgeWeb (EU Network of Excellence IST-2004-507482), TAO (EU FP6 project IST-2004-026460), SEKT (EU FP6 project IST-IP-2003-506826, Líon (Science Foundation Ireland project SFI/02/CE1/1131) and NEPOMUK (EU project FP6-027705).

\section{References}

1. Funk, A., Tablan, V., Bontcheva, K., Cunningham, H., Davis, B., Handschuh, S.: Clone: Controlled language for ontology editing. In: ASWC 2007 and ISWC 2007. LNCS, vol. 4825, pp. 142-155. Springer, Heidelberg (2007)

2. Cunningham, H., Maynard, D., Bontcheva, K., Tablan, V.: GATE: A Framework and Graphical Development Environment for Robust NLP Tools and Applications. In: Proceedings of the 40th Anniversary Meeting of the Association for Computational Linguistics (ACL 2002) (2002)

3. Thompson, C.W., Pazandak, P., Tennant, H.R.: Talk to your semantic web. IEEE Internet Computing 9(6), 75-78 (2005)

4. Funk, A., Davis, B., Tablan, V., Bontcheva, K., Cunningham, H.: Controlled language IE components version 2. Deliverable D2.2.2, SEKT (2006)

5. Noy, N.F., McGuinness, D.L.: Ontology development 101: A guide to creating your first ontology. Technical Report KSL-01-05, Stanford Knowledge Systems Laboratory (March 2001)

6. Brooke, J.: SUS: a "quick and dirty" usability scale. In: Jordan, P., Thomas, B., Weerdmeester, B., McClelland, A. (eds.) Usability Evaluation in Industry, Taylor and Francis, London (1996)

7. Tullis, T.S., Stetson, J.N.: A comparison of questionnaires for assessing website usability. In: Usability Professionals' Association Conference, Minneapolis, Minnesota (June 2004)

\footnotetext{
17 http://nepomuk.semanticdesktop.org/xwiki/

18 http://nepomuk-kde.semanticdesktop.org/xwiki/bin/view/Main/WebHome

19 http://smile.deri.ie/projects/semn/

20 http://knowledgeweb.semanticweb.org/
} 
8. Bailey, B.: Getting the complete picture with usability testing. Usability updates newsletter, U.S. Department of Health and Human Services (March 2006)

9. Phillips, J.L.: How to Think about Statistics. W.H. Freeman and Company, New York (1996)

10. Connolly, T.G., Sluckin, W.: An Introduction to Statistics for the Social Sciences, 3rd edn. Macmillan, Basingstoke (1971)

11. Schwitter, R.: Controlled natural languages. Technical report, Centre for Language Technology, Macquarie University (June 2007)

12. Adriaens, G., Schreurs, D.: From COGRAM to ALCOGRAM: Toward a controlled English grammar checker. In: Conference on Computational Linguistics (COLING 1992), Nantes, France, pp. 595-601 (1992)

13. Fuchs, N., Schwitter, R.: Attempto Controlled English (ACE). In: CLAW 1996. Proceedings of the First International Workshop on Controlled Language Applications, Leuven, Belgium (1996)

14. Kaljurand, K., Fuchs, N.E.: Bidirectional mapping between OWL DL and Attempto Controlled English. In: Fourth Workshop on Principles and Practice of Semantic Web Reasoning, Budva, Montenegro (June 2006)

15. Kuhn, T.: Attempto Controlled English as ontology language. In: Bry, F., Schwertel, U. (eds.) REWERSE Annual Meeting 2006 (March 2006)

16. Kaljurand, K., Fuchs, N.: Verbalizing OWL in Attempto Controlled English. In: Proceedings of OWL: Experiences and Directions, OWLED 2007 (2007)

17. Piwek, P.: Requirements definition, validation, verification and evaluation of the clime interface and language processing technology. Technical report, ITRIUniversity of Brighton (2002)

18. Bernstein, A., Kaufmann, E.: GINO - a guided input natural language ontology editor. In: Cruz, I., Decker, S., Allemang, D., Preist, C., Schwabe, D., Mika, P., Uschold, M., Aroyo, L.M. (eds.) ISWC 2006. LNCS, vol. 4273. Springer, Heidelberg (2006)

19. Namgoong, H., Kim, H.: Ontology-based controlled natural language editor using cfg with lexical dependency. In: Aberer, K., Choi, K.-S., Noy, N., Allemang, D., Lee, K.-I., Nixon, L., Golbeck, J., Mika, P., Maynard, D., Mizoguchi, R., Schreiber, G., Cudré-Mauroux, P. (eds.) ASWC 2007 and ISWC 2007. LNCS, vol. 4825, pp. 353-366. Springer, Heidelberg (2007)

20. Lopez, V., Motta, E., Uren, V.: Poweraqua: Fishing the semantic web. In: Sure, Y., Domingue, J. (eds.) ESWC 2006. LNCS, vol. 4011, pp. 393-410. Springer, Heidelberg (2006) 